\title{
Water Effects on the First-Order Transition in a Model of Earthquakes
}

\author{
M. W. Dongmo, L. Y. Kagho, F. B. Pelap, G. B. Tanekou, Y. L. Makenne, and A. Fomethe \\ Laboratory of Mechanics and Modelling of Physical Systems, Department of Physics, University of Dschang, \\ P.O. Box 69, Dschang, Cameroon
}

Correspondence should be addressed to M. W. Dongmo; mathurin.wamba@yahoo.fr

Received 15 November 2013; Accepted 21 January 2014; Published 16 March 2014

Academic Editors: E. Del Pezzo, E. Liu, and A. Stovas

Copyright (c) 2014 M. W. Dongmo et al. This is an open access article distributed under the Creative Commons Attribution License, which permits unrestricted use, distribution, and reproduction in any medium, provided the original work is properly cited.

\begin{abstract}
The study of 1D spring-block model of earthquake dynamics with consideration of water effects in preexisting fault deals with new forms of frictional force. An analytical study of the equation of motion enables us to establish that motion of geological fault is accelerated by water pressure. In the same setting the critical value of frictional velocity for which appears the discontinuous (firstorder) transition from a stick-slip behavior to a creep motion strongly depends on water pressure. The investigation also displays the magnitude and probability of events as a function of water pressure; these two quantities decrease and increase, respectively, with the variation of water pressure.
\end{abstract}

\section{Introduction}

Despite significant advances made in the study of geological fault structures and plate tectonics, our understanding of the physical mechanisms responsible for the initiation, propagation, and termination of earthquake rupture remains unfinished. Burridge and Knopoff [1], in 1967, introduced a one-dimensional chain block and spring-discrete model, aiming at explaining the earthquake mechanism [2]. In 1996, Vasconcelos simplified Knopoff'smodel with a single springblock model and attempted to facilitate the understanding of earthquake using this model. The mechanism of slip instabilities in laboratory experiments has been proposed to be dependent on several factors including reduced frictional force during sliding (slip weakening) and a decrease in slip velocity [3], which is in concordance with the observation of the geologist Rick Sibson [4], who in 1981 maintained the idea affirming that water pressure in the fault was opposite to the rocks' pressure, which intensified friction between the fault's sides. The mathematical model which takes into consideration this idea was not yet established; however, some studies have been done in dry faults with the help of wellknown mathematical models $[5,6]$. Moreover, Vasconcelos [6] had investigated the phase transition in this single block model and demonstrated that it occurs (from stick-slip to creep motion) only when the characteristic velocity is equal to 0.5 . This result was obtained because he considered dry fault in his study. In other cases there are several values of characteristic speed (less than 0.5 ) which are able to lead to the transition.

The aim of this paper is to study Giovanni's modified single block model, by considering the new shape of frictional forces (that contains water pressure), and investigate the effects of water pressure on the earthquake dynamics. The content of this paper is organized as follows.

Section 2: frictional model and water effects.

Section 3: equation of motion and temporal evolution of displacements.

Section 4: effects of pressure on the first-order transition.

Section 5: effects of pressure on the magnitude and the probability of occurrence of an event.

The last section is devoted to discussion and conclusion.

\section{Description of Water Effects in the Fault and Equation of Motion}

Figure 1(a) displays a preexisting fault broken on one side and impervious at the other side. Water gets into the fault 


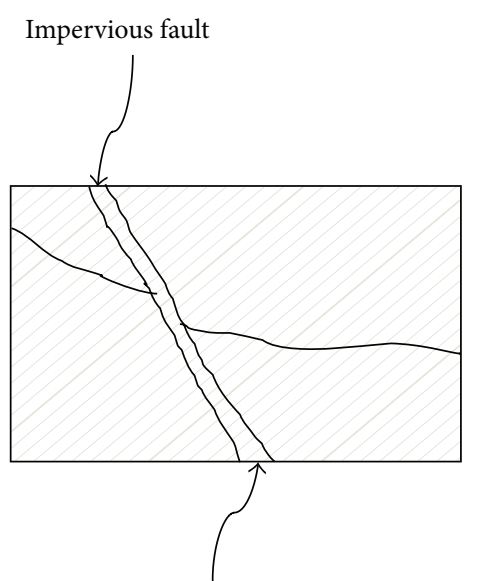

Fractured and permeable fault

(a)

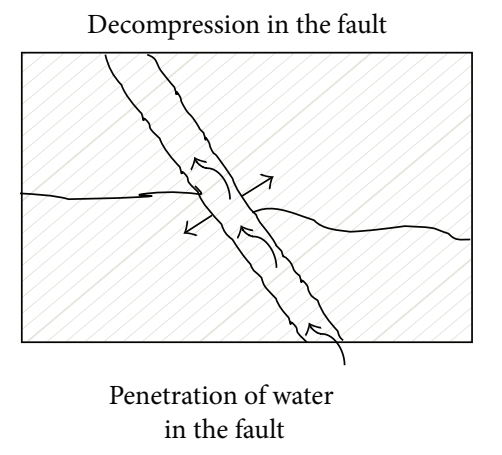

(b)

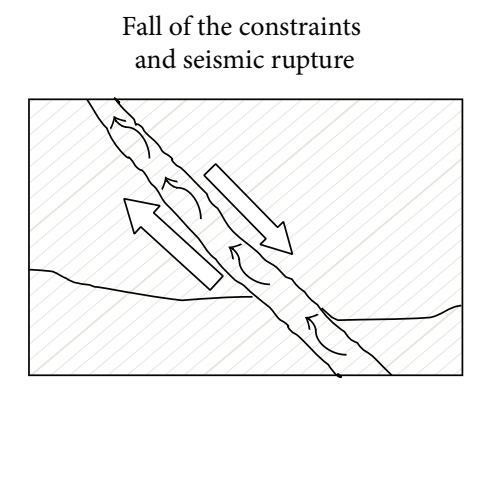

(c)

Figure 1: (a) Fault with permeable side and impervious side. (b) Very high pressure of water. (c) Flux of water in the fault.

through the fractured side; it thwarts the pressure of the rocks which intensifies friction between the two sides of the fault (Figure 1(b)). This will provoke a seismic rupture when water pressure becomes increasingly intensified [4] (Figure 1(c)).

Since our investigation focused on the role of water on the fault's dynamics, we hereby propose a new form of frictional force that takes into consideration water characteristic in the process. The model below has a great advantage of being analytically tractable, so that one hopes that a thorough understanding of such a simple model might in turn shed further light on the basic principles governing real earthquakes. We will for convenience choose the frictional force such as $f(d X / d t)=F_{0} \Phi\left(\left(1 / V_{f}\right)(d X / d t)\right)-\Gamma_{0} \Phi\left(\left(1 / V_{f}\right)(d X / d t)\right)$, where $F_{0}$ corresponds to the maximum frictional force (greater than $\Gamma_{0}$ ).

$\Gamma_{0}=\rho S V_{e}^{2} / 2$ is the strength of pressure, $\rho$ is the density of water, $S$ is the surface of the fault, and $V_{e}$ is the speed of water in the fault assumed to vary weakly, such as a constant in our equation. The quantities $(d X / d t)$ and $V_{f}$ represent the velocity of the fault and the characteristic velocity for the friction, respectively.

$\Phi(x)$ is assumed to be a continuous function for $x \geq 0$ satisfying the initial conditions $\Phi(0)=1$ and $(d \Phi / d x)_{x=0}=-1$; the second conditions simply express the velocity-weakening effect of the friction, since it implies that $f(d X / d t)$ will be a decreasing function of the block velocity $d X / d t$, at least in a neighborhood of origin. In this model (Figure 1), a block of mass $m$ is connected by spring of constant $K$ (corresponding to the linear elastic properties of the medium surrounding the fault) to a rigid pulling rod that moves at a small constant velocity $V$ (Figure 2). The block rests upon a stationary surface, which provides a velocity dependent on frictional force $f$, which impedes the motion of the block. It is important to note that water pressure acts between the block and the stationary surface, such that it reduces the frictional force $f$.
When the force due to the spring exceeds the threshold force denoted by $F_{0}-\Gamma_{0}$, the block is set into motion and the corresponding equation of motion is

$$
m \frac{d^{2} X}{d t^{2}}=K(V t-X)-f\left(\frac{d X}{d t}\right)
$$

where $X(t)$ is the position of the block. Owing to the velocityweakening effect of friction, the block undergoes a rapid motion (earthquakes), during which most of the accumulated stress is released. Before proceeding with the analysis, it is convenient to introduce dimensionless variables:

$$
U=\left(\frac{K}{F_{0}}\right) X, \quad \tau=\left[\frac{K}{m}\right]^{1 / 2} t .
$$

So that the equation of motion (1) takes the dimensionless form

$$
\frac{d^{2} U}{d \tau^{2}}=-U+\nu \tau+\left(-1+\frac{\Gamma_{0}}{F_{0}}\right) \Phi\left(\frac{1}{v_{f}} \frac{d U}{d \tau}\right)
$$

with $\nu=V / V_{0}, v_{f}=V_{f} / V_{0}$, and $v_{e}=V_{e} / V_{0}, \mu_{e}=\rho S\left(V_{0}^{2} / F_{0}\right)$, $p_{e}=\mu_{e} v_{e}^{2} / 2$, and $V_{0}=F_{0} / \sqrt{m K}$. The velocity scale $V_{0}$ corresponds to the maximum velocity attained by a block that experiences no (kinematic) friction as it moves. The dimensionless parameters $v$ and $v_{f}$ are, respectively, the pulling speed and friction characteristic velocity measured in this scale. In the same manner $\mu_{e}, p_{e}, v_{e}$ are, respectively, the density, the pressure, and the velocity of water in the same scale.

For this model to be relevant for real earthquakes, dimensionless water pressure must be less than one (i.e., $p_{e}<$ 1 ; we recall that $\Gamma_{0}<F_{0}$ ) and $\nu$ must be taken to be very small. Indeed, during an earthquake the relative velocity between the two sides of the fault is of the order $\mathrm{m} / \mathrm{s}$, while the typical relative plate velocity is of the order $\mathrm{cm} / \mathrm{yr}$. 


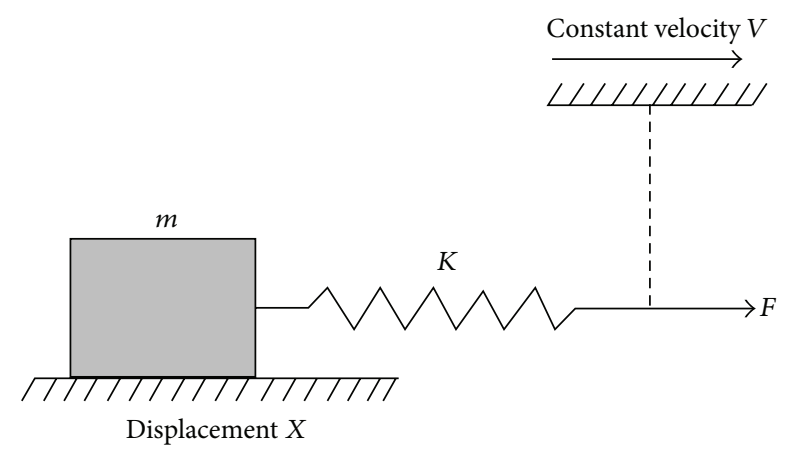

FIGURE 2: Spring-block model for earthquakes.

We start the analysis by considering first the linearized version of the motion equation. In view of (1) and (2) the linearization of (3) yields

$$
\frac{d^{2} U}{d \tau^{2}}-2 \alpha\left(1-p_{e}\right) \frac{d U}{d \tau}+U=v \tau .
$$

For convenience we have introduced the parameter $\alpha=$ $1 / 2 \nu_{f}$ and $\gamma=\alpha\left(1-p_{e}\right)$, with $0 \leq p_{e}<1$. After this simplification and redefinition of the origin of displacement so as to eliminate the constant that would otherwise appear on the right-hand side of (4), we have

$$
\frac{d^{2} U}{d \tau^{2}}-2 \gamma \frac{d U}{d \tau}+U=\nu \tau .
$$

The linear approximation above will be valid only if the block velocity is small compared to the friction characteristic velocity (i.e., $\left.(d U / d \tau) \ll v_{f}\right)$. The initial conditions are given by $\left(d^{2} U(0) / d \tau^{2}\right)=(d U(0) / d \tau)=U(0)=0$.

Equation (5) presents two cases to be considered: (i) $v_{f}>$ $\left(1-p_{e}\right) / 2$ and (ii) $v_{f} \leq\left(1-p_{e}\right) / 2$.

\section{Oscillatory Solutions}

Now, our attention is focused on the creep motion of the block; for this we consider the case (i) and then the solution of (5) is

$$
\begin{gathered}
U(\tau)=\nu\left[\exp (\gamma \tau)\left(\frac{2 \gamma^{2}-1}{\omega} \sin \omega \tau-2 \gamma \cos \omega \tau\right)\right. \\
+\tau+2 \gamma],
\end{gathered}
$$

where $\omega=\sqrt{1-\gamma^{2}}$. The velocity of block is obtained by time derivative of displacement of the block. From (6) we have

$$
\dot{U}(\tau)=\nu\left[\left(\frac{\gamma}{\omega} \sin \omega \tau-\cos \omega \tau\right) \exp (\gamma \tau)+1\right],
$$

where the dots indicate time derivatives; the maximum velocity attained by the block is

$$
\dot{U}_{\max }=v\left[1+\exp \left(\frac{\gamma \pi}{\omega}\right)\right] .
$$

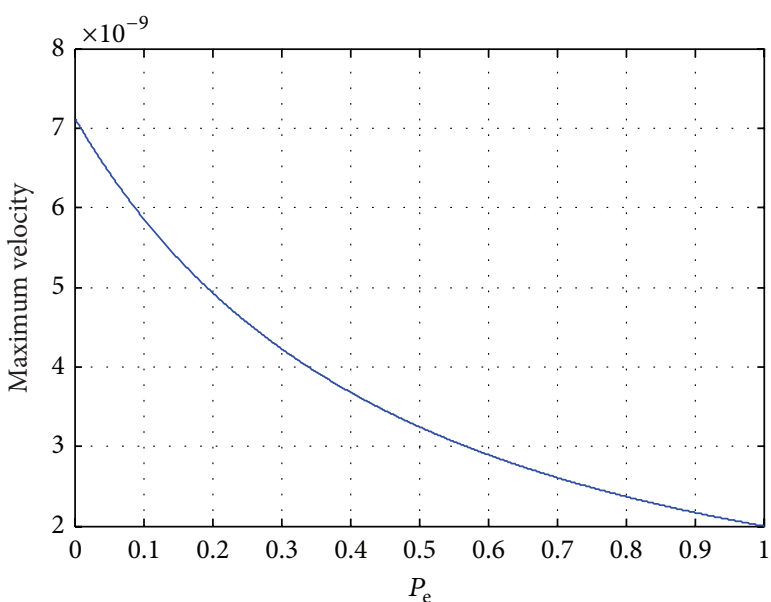

FIGURE 3: Effects of water pressure on the magnitude of maximum velocity $\left(\nu=10^{-9}\right.$ and $\left.\alpha=0.5\right)$.

It appears from Figure 3 that water pressure strongly affects the magnitude of maximum velocity attained by the block; at high pressures, the block rapidly reaches its cruise velocity and thus it can be easily understood that the fault's motion is accelerated by water pressure.

During the description of earthquake, it is important to define the position of the block at the end of the slip. But, when the block ceases to move, it occupies an unspecified position. However, determination of this position requires the knowledge of the time $\tau_{0}$ at which the block ceased to slip. Concretely, when the block stops, its speed vanishes. Therefore, the corresponding time $\tau_{0}$ is the solution of the following equation:

$$
\frac{\gamma}{\omega} \sin \omega \tau_{0}-\cos \omega \tau_{0}+\exp \left(-\gamma \tau_{0}\right)=0
$$

Substituting (9) into (6) we notice that the block's displacement $\Delta=U\left(\tau_{0}\right)$ after such slip event is given by

$$
\Delta=U\left(\tau_{0}\right)=\nu\left[\tau_{0}+\gamma+\sqrt{\exp \left(2 \gamma \tau_{0}\right)-\omega^{2}}\right] .
$$

Since $\tau_{0}$ does not depend on $\nu$, it then follows that as $v \rightarrow 0$ the displacement $\Delta$ vanishes whenever $v_{f}>\left(1-p_{e}\right) / 2$.

Next we investigate the situation when $v_{f} \leq\left(1-p_{e}\right) / 2$.

\section{A Periodic Solution of the Motion Equation}

The main objective of this section is to investigate the behavior of the block in stick-slip motion. Considering the case (ii): $v_{f} \leq\left(1-p_{e}\right) / 2$, the solution of (5) is given by

$$
\begin{gathered}
U(\tau)=\nu\left[\left(\frac{2 \gamma^{2}-1}{\omega} \sinh \omega \tau-2 \gamma \cosh \omega \tau\right)\right. \\
\quad \times \exp (\gamma \tau)+\tau+2 \gamma]
\end{gathered}
$$

with $\omega=\sqrt{\gamma^{2}-1}$. 


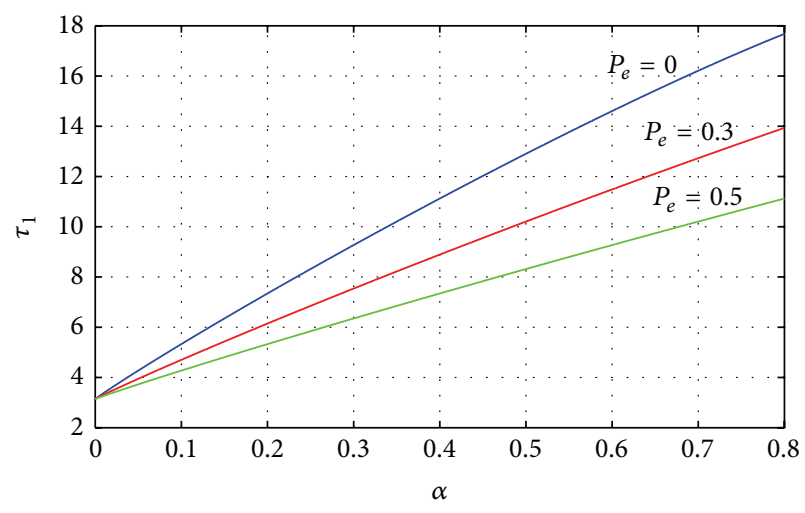

(a)

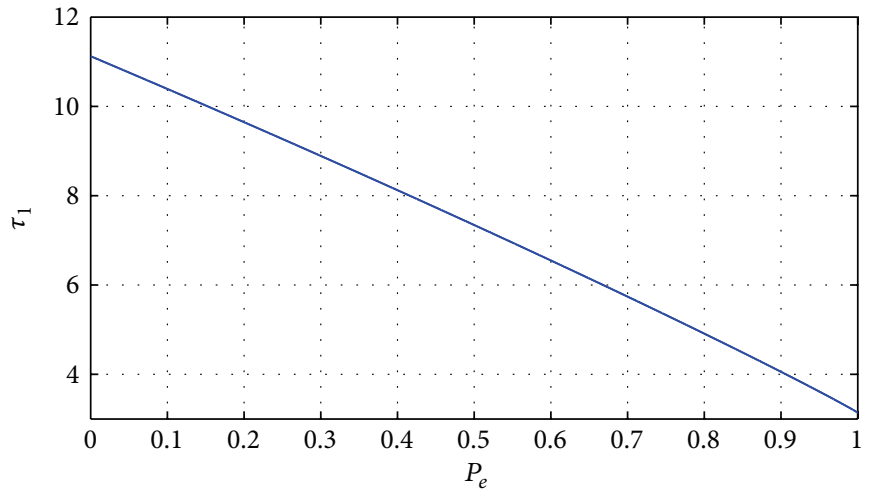

(b)

Figure 4: (a) Effects of water pressure on the closed time of linear motion part for $\left(p_{e}=0, p_{e}=0.3, p_{e}=0.5\right)$. (b) Closed time of linear part of motion versus water pressure (with $\alpha=0.4$ ).

Since the velocity $d U / d \tau$ is now a monotonously increasing function of time, the block eventually reaches a velocity comparable to the characteristic velocity $v_{f}$ of the friction, independently of the smallness of $v$. Around such point, the linear approximation is useless since the motion of the block becomes nonlinear. It is thus necessary to consider another model of the friction law which will not present such behavior.

Several friction models have been recently considered in the literature $[7,8]$. Hereafter, we consider the simple model proposed by Langer and Tang [7] to examine the behavior of our system; that is,

$$
\Phi(x)= \begin{cases}1-x, & \text { if } 0 \leq x \leq 1 \\ 0, & \text { if } x \geq 1\end{cases}
$$

With this model, the motion equation (5) remains valid and presents two distinguished cases which correspond, respectively, to (i) and (ii). We recall that if $\nu_{f}>\left(1-p_{e}\right) / 2$, then $\Delta \rightarrow 0$ as $v \rightarrow 0$, regardless of the nonlinear features of the friction law. In this case, the solution for the block motion can be divided into three parts [6] as follows.

Initially, when $(d U / d \tau)<v_{f}$ the motion of the block is confined to the linear part of the friction and hence the solution of (5) is given by (11). This solution is valid until the time $\tau_{1}$, where $\left(d U\left(\tau_{1}\right) / d \tau\right)=v_{f}$, after which the block enters the nonlinear regime of the friction law. In the limit $v \rightarrow 0$, this time $\tau_{1}$ diverges logarithmically with the term $v$ :

$$
\tau_{1}=(\gamma-\omega) \ln \left[\frac{\omega(\omega+\gamma)}{\gamma \nu}\right] .
$$

We examine here the influence of the pressure on the time $\tau_{1}$ marking the onset of nonlinear motion.

Figures 4(a) and 4(b) show, respectively, the reduction of $\tau_{1}$ (as function of $\alpha$ ) for the greater values of the pressures and decreasing of $\tau_{1}$ as a function of pressure for the fixed value of $\alpha$.

From here it is easy to understand that water can considerably reduce the time of transition (from a stick-slip behavior to a creep motion). Although the block spends a very long time in this linear regime, considerable motion will occur only for times close to the instant $\tau_{1}$. Therefore, it is convenient to introduce the renormalized time $t=\tau-\tau_{1}$. The block position in terms of $t$ can now be obtained by substituting (13) into (11) and taking the limit $\nu \rightarrow 0$. After some simplifications, we find that

$$
U(t)=v_{f}(\gamma-\omega) \exp (\gamma+\omega) t
$$

For time $t>0$, the motion consists of two parts [6]. First, the block will swing frictonlessly until the time $t_{1}$ (defined below) at which its velocity is again equal to $v_{f}$. Afterwards, the block experiences once more a nonzero (linear) friction until it finally stops later at the time $t_{2}$. Since we are interested in the linear behavior of the system in the limit $v \rightarrow 0$, we set $v=0$ in (5) and exploit the Langer and Tang model [7] to examine the dynamics of the block in these two regions. Computations lead to the complete solution of the motion equation that defines the position of the block for time $>0$ :

$$
\begin{aligned}
& U(t) \\
& =\left\{\begin{array}{cc}
v_{f}[\sin t-(\gamma+\omega) \cos t], & \text { if } 0 \leq t \leq t_{1}, \\
v_{f} e^{\gamma\left(t-t_{1}\right)}\left[(3 \gamma+\omega) \cosh \omega\left(t-t_{1}\right)\right. & \\
-\left(\gamma+3 \omega+\left(\frac{2}{\omega}\right)\right) & \\
\left.\times \sinh \omega\left(t-t_{1}\right)\right], & \text { if } t_{1} \leq t \leq t_{2},
\end{array}\right.
\end{aligned}
$$

in which explicit expressions of the times $t_{1}$ and $t_{2}$ are

$$
t_{1}=2 \arctan (\gamma+\omega) ; \quad t_{2}=t_{1}+\frac{1}{2 \omega} \ln \left(1+\frac{\omega}{\gamma}\right)
$$




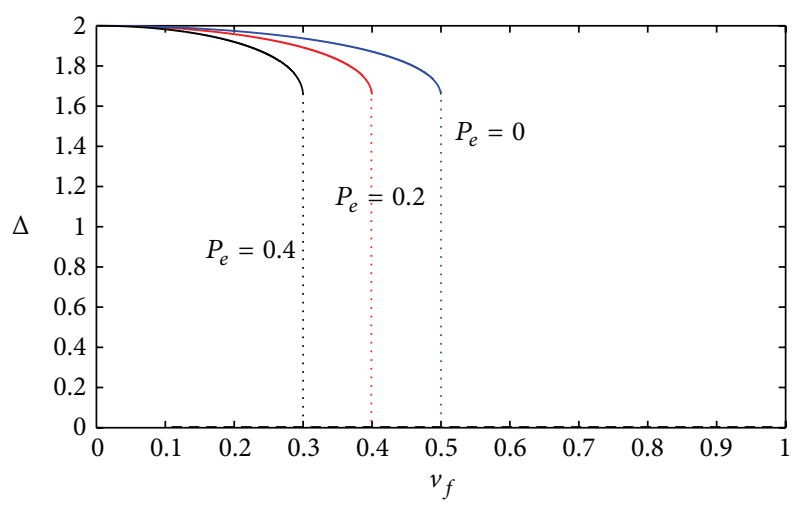

FIGURE 5: The block displacement $\Delta$ versus the friction characteristic $v_{f}$ for three values of water pressure in model (12).

After the earthquake, the position of the block (fault's displacement) is obtained by evaluating the quantity $\Delta=U\left(t_{2}\right)$; that is,

$$
\Delta= \begin{cases}\left(1+\frac{\omega}{\gamma}\right)^{(1 / 2)(1+(\gamma / \omega))}, & \text { if } v_{f} \leq \frac{\left(1-p_{e}\right)}{2} \\ 0, & \text { if } v_{f}>\frac{\left(1-p_{e}\right)}{2} .\end{cases}
$$

Here, we also collect the aforementioned results that $\Delta$ vanish for $v_{f}>\left(1-p_{e}\right) / 2$ (with respect to $v \rightarrow 0$ ). Then, we note that at $v_{f}=\left(1-p_{e}\right) / 2$, the system undergoes a phase transition in the sense that $\Delta$ vanishes for $v_{f}>\left(1-p_{e}\right) / 2$ while it takes finite values for $v_{f} \leq\left(1-p_{e}\right) / 2$.

A curve given the evolution of $\Delta$ versus $v_{f}$ for several values of $p_{e}$ is plotted in Figure 5.

The curve of Figure 5 exhibits that water pressure has a remarkable effect on $\Delta$ (fault's displacement) at the end of sliding, whereof it is easy to note that the first-order transition depends on the value of water pressure. The transition occurs increasingly with increase in pressure; thus a fast transition allows for short-period earthquake. Worthy of note is the fact that $p_{e}=0$ corresponds to the dry fault studied in [6]. Actually the system undergoes a discontinuous (first-order) transition from stick-slip behavior to creep motion as the friction parameter is varied.

Following the study of water effects on the first-order transition, it is worthy to investigate the influence of water on energy release during an earthquake.

\section{Magnitude of Earthquake and Probability of Occurrence}

The objective of this section is to investigate the effects of water pressure on the earthquake magnitude. Several magnitude expressions have been investigated. Some of these take into consideration the parameters characterizing the seismic wave [9-11], and others consider the physical parameter of the rocks $[12,13]$.
Hereafter, we consider the magnitude expression formulated by Kanamori et al. [14, 15]:

$$
M=\frac{2}{3}\left[\log _{10}\left(\frac{M_{0}}{1 N \cdot m}\right)-9.1\right],
$$

where the seismic moment is

$$
M_{0}=\mu S \Delta .
$$

See $[16,17]$.

Wherein $\mu$ defines the rocks rigidity, $S$ represents the fractured surface and $\Delta$ the fault's displacement. Owing to the fact that the surface $S$ is proportional to the length of the fault which is itself proportional to the position $\Delta$, the seismic moment (19) takes the form [4]

$$
M_{0}=K_{0} \Delta^{3} .
$$

Substituting (20) and (19) into (18), then (18) becomes

$$
= \begin{cases}\frac{3}{2}\left[\log _{10}\left(K_{0}\left(1+\frac{\omega}{\gamma}\right)^{(3 / 2)(1+(\gamma / \omega))}\right)-9.1\right], & \text { if } v_{f} \leq \frac{\left(1-p_{e}\right)}{2}, \\ \text { not defined, } & \text { if } v_{f}>\frac{\left(1-p_{e}\right)}{2} .\end{cases}
$$

In (20), $K_{0}$ is a constant which can be determined by exploiting the experimental curve giving the magnitude as a function of displacement in [4], $\left(K_{0} \sim 1.2589254 .10^{18}\right)$.

The effect of water on the magnitude is investigated by plotting the magnitude $M$ as function of pressure $p_{e}$ (Figure 6).

From Figure 6 we notice that the magnitude decreases logarithmically with the pressure until it reaches its smallest value, at $p_{e}=0.75$ (corresponding to the transition point for $\left.v_{f}=0.125\right)$. Note equally in Figure 6 that when $p_{e} \rightarrow 0$ the magnitude has a maximum value. When $p_{e} \geq 0.75$, the magnitude is not defined because the logarithm of zero does not exist. Recall that $p_{e}=0.75$ corresponds to the value for which the phase transition occurs (i.e., when $\Delta$ vanishes). It is important to remark that the critical value of $p_{e}$ from which the magnitude is not defined changes with fixed values of $v_{f}$. It arises from this curve (Figure 6) that the magnitude of the earthquake decreases when the pressure of water in geological fault increases; from here it is important to note that when water gets into the fault, there is an impact on the energy release during its sliding.

Now let us examine the influence of pressure on the probability of earthquakes occurrence; as defined by Gutenberg and Richter in 1944 [18] it is

$$
W=10^{\left(c-d \times \log M_{0}\right)} .
$$

In this previous quantity, $M_{0}$ is defined by (20); after substitution of (20) into (22) we have

$$
W=10^{c-d \times \log \left(K_{0} \cdot \Delta^{3}\right)},
$$

where $c$ and $d$ are arbitrary constants. 


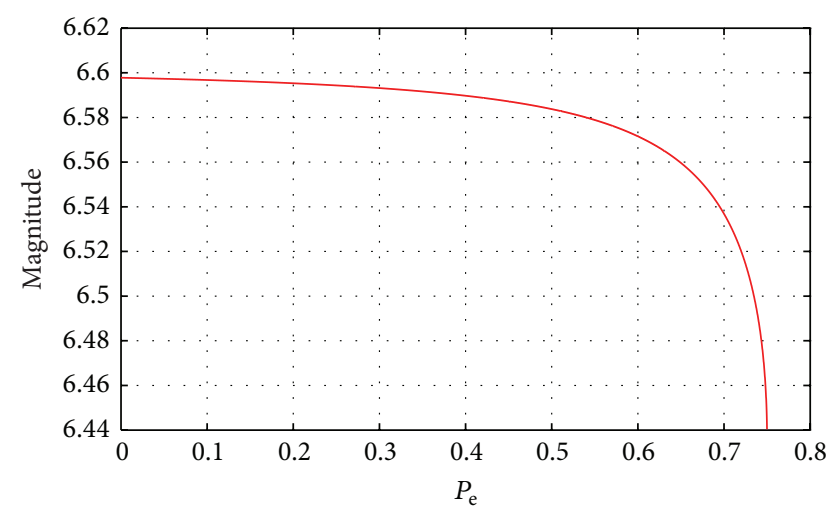

FIGURE 6: Magnitude $M$ versus the pressure of water $p_{e}$ (with $v_{f}=$ $0.125)$.

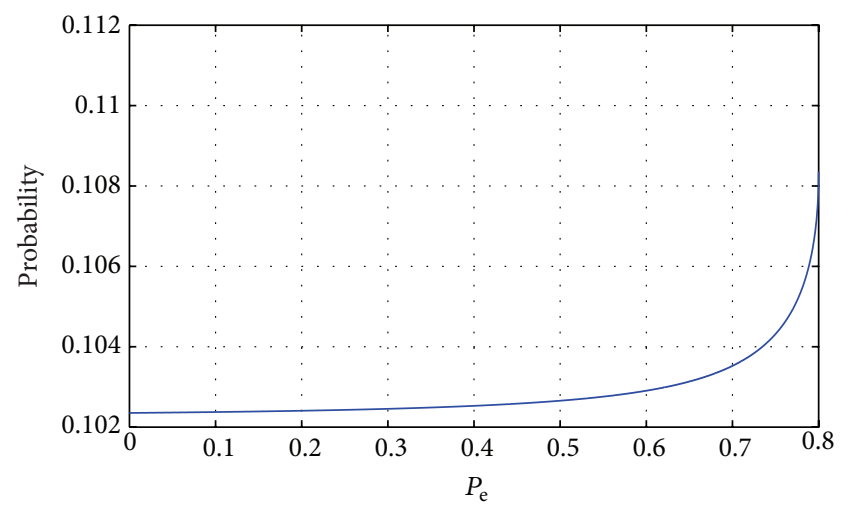

FIGURE 7: Probability of events in terms of water pressure with $v_{f}=$ $0.1 ; c=0.91$; and $d=0.1$.

It appears from Figure 7 that the probability of occurrence of an event increases with water pressure. So this result is in accordance with the results obtained in Figures 4 and 5, because the closed time of linear motion decays with pressure, and the transition occurs rapidly with greater values of pressure. This means that water in the fault can cause more earthquakes with smaller magnitude (precisely for $p_{e}<1$ ).

It is important to remark that the value of frictional velocity which leads to the transition at $p_{e}=0.8$ is $\nu_{f}=0.1$.

\section{Conclusion}

In this paper, we have studied water effects on the dynamics of a 1D spring-block model for earthquakes by performing the Vasconcelos frictional force [6]. We have shown that the system undergoes a discontinuous phase transition from a stick-slip motion to a creep motion as the frictional parameter varies. We established that this transition is not dependent only on the greatness of the driving plate's velocity (great improvement of the Vasconcelos' results [6]) but depends also on the value of water pressure in the fault; contrary to Vasconcelos we revealed that the value of characteristic frictional velocity which leads to the transition is not unique (0.5) but that it varies according to the value of water pressure in the fault. Through our investigation, it is established that for the greater values (less than one) of water pressure the transition occurs rapidly (i.e., short-period earthquake) as compared to the smallest or zero pressure. These high pressures allow for weaker magnitude earthquakes. Thus the propagation of water in the fault could be of an advantage as it permits the occurrence of earthquakes with lower magnitude. In general, more events will be recorded (with weak magnitude) in faults containing water than in dry ones.

\section{Conflict of Interests}

The authors do not have any conflict of interests regarding the publication of this paper.

\section{Acknowledgment}

Professor F. B. Pelap is grateful to Professor Giovani L. Vasconcelos for valuable discussions during his last visit in the University of Pernembuco, Brazil.

\section{References}

[1] R. Burridge and L. Knopoff, "Model and theoretical seismicity," Bulletin of the Seismological Society of America, vol. 57, pp. 341371, 1967.

[2] J. M. Carlson and J. S. Langer, "Properties of earthquakes generated by fault dynamics," Physical Review Letters, vol. 62, no. 22, pp. 2631-2635, 1989.

[3] A. Ruina, "Slip instability and state variable friction laws," Journal of Geophysical Research, vol. 88, no. 12, pp. 10359-10370, 1983.

[4] P. Bernard, “Qu'est-ce qui fait trembler la terre?” EDP Science, 2003.

[5] R. Montagne and G. L. Vasconcelos, "Complex dynamics in a one-block model for earthquakes," Physica A, vol. 342, no. 1-2, pp. 178-185, 2004.

[6] G. L. Vasconcelos, "First-order transition in a model of earthquakes," Physical Review Letters, vol. 76, p. 25, 1996.

[7] J. S. Langer and C. Tang, "Rupture propagation in a model of an earthquake fault," Physical Review Letters, vol. 67, no. 8, pp. 1043-1046, 1991.

[8] J. M. Carlson and J. S. Langer, "Mechanical model of an earthquake fault," Physical Review Letters, vol. 62, p. 2632, 1989.

[9] C. F. Richter, "An instrumental earthquake magnitude scale," Bulletin of the Seismological Society of America, vol. 25, pp. 132, 1935.

[10] B. Gutenberg, "Amplitudes of surface waves and magnitude of shallow earthquakes," Bulletin of the Seismological Society of America, vol. 35, pp. 3-12, 1945.

[11] B. Gutenberg and C. F. Richter, "Earthquake magnitude, intensity, energy, and acceleration," Bulletin of the Seismological Society of America, vol. 46, pp. 105-146, 1956.

[12] H. Kanamori, “The energy release in great earthquakes," Journal of Geophysical Research, pp. 2981-2987, 1977.

[13] T. C. Hanks and H. Kanamori, "A moment magnitude scale," Journal of Geophysical Research B, vol. 84, no. 5, pp. 2348-2350, 1979. 
[14] H. Kanamori and D. Hadley, "Crustal structure and temporal velocity change in southern California," Pure and Applied Geophysics, vol. 113, no. 1, pp. 257-280, 1975.

[15] H. Kanamori, H.-K. Thio, D. Dreger, E. Hauksson, and T. Heaton, "Initial investigation of the Landers, California, earthquake of 28 June 1992 using TERRAscope," Geophysical Research Letters, vol. 19, no. 22, pp. 2267-2270, 1992.

[16] K. Aki, "Generation and propagation of G waves from the Niigata Earthquake of June 16, 1964: Part 1. A statistical analysis," Bulletin of the Earthquake Research Institute, vol. 44, pp. 23-72, 1966.

[17] J. Vaneck, A. Zapotek, V. Karnik et al., "Standardization of magnitude scales," Izvestiya Akademii Nauk SSSR Seriya Geofizicheskii, pp. 153-158, 1962.

[18] B. Gutenberg and C. F. Richter, "Frequency of earthquakes in California," Bulletin of the Seismological Society of America, vol. 34, pp. 185-188, 1944. 

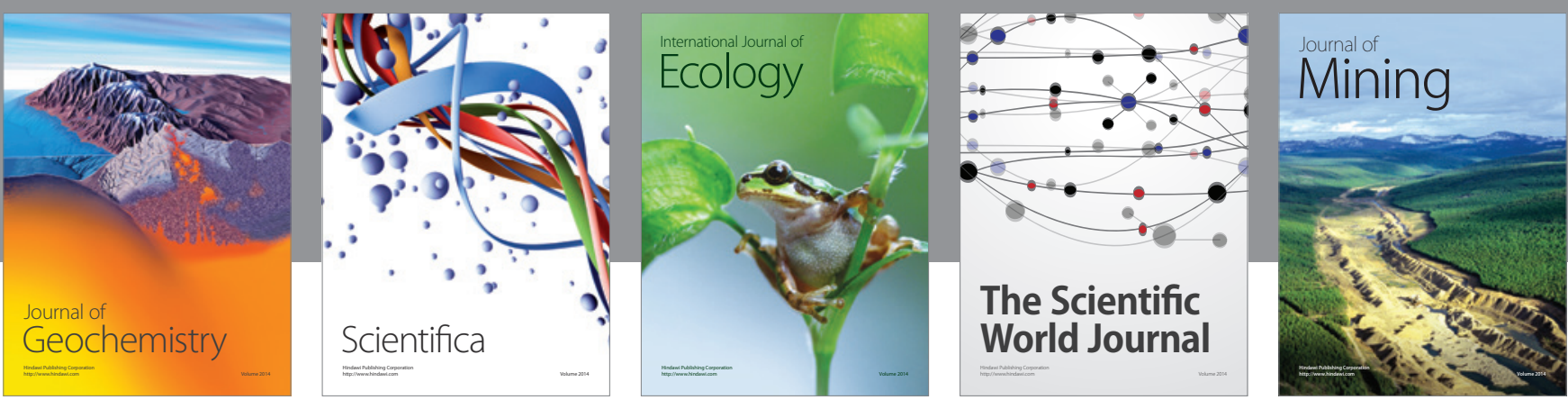

The Scientific World Journal
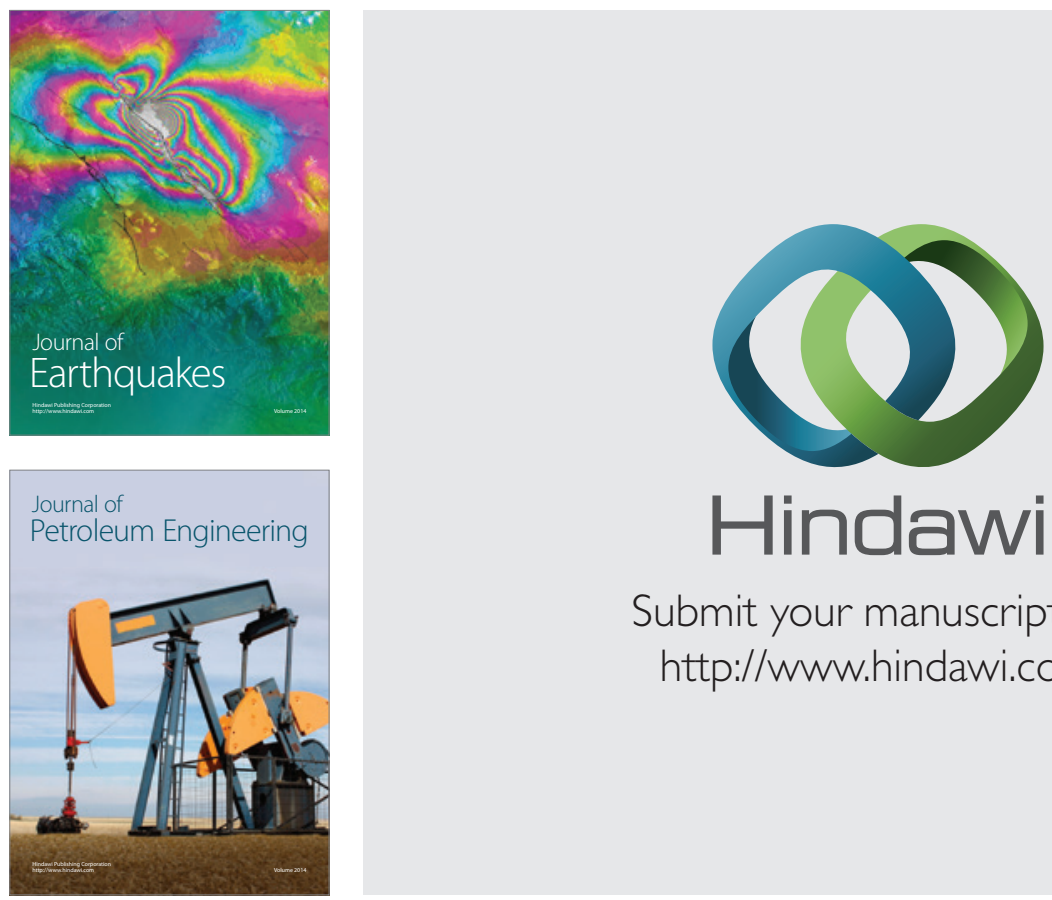

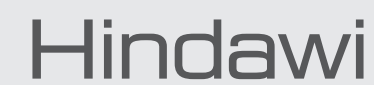

Submit your manuscripts at

http://www.hindawi.com
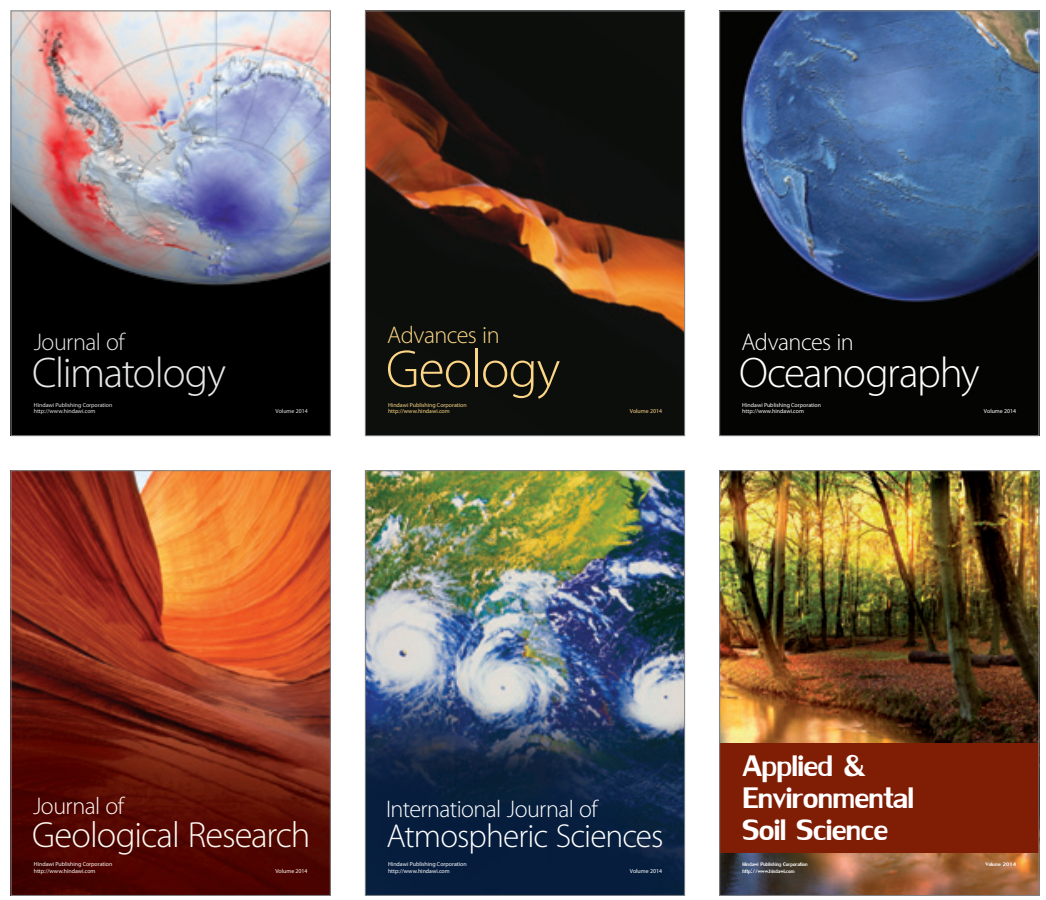
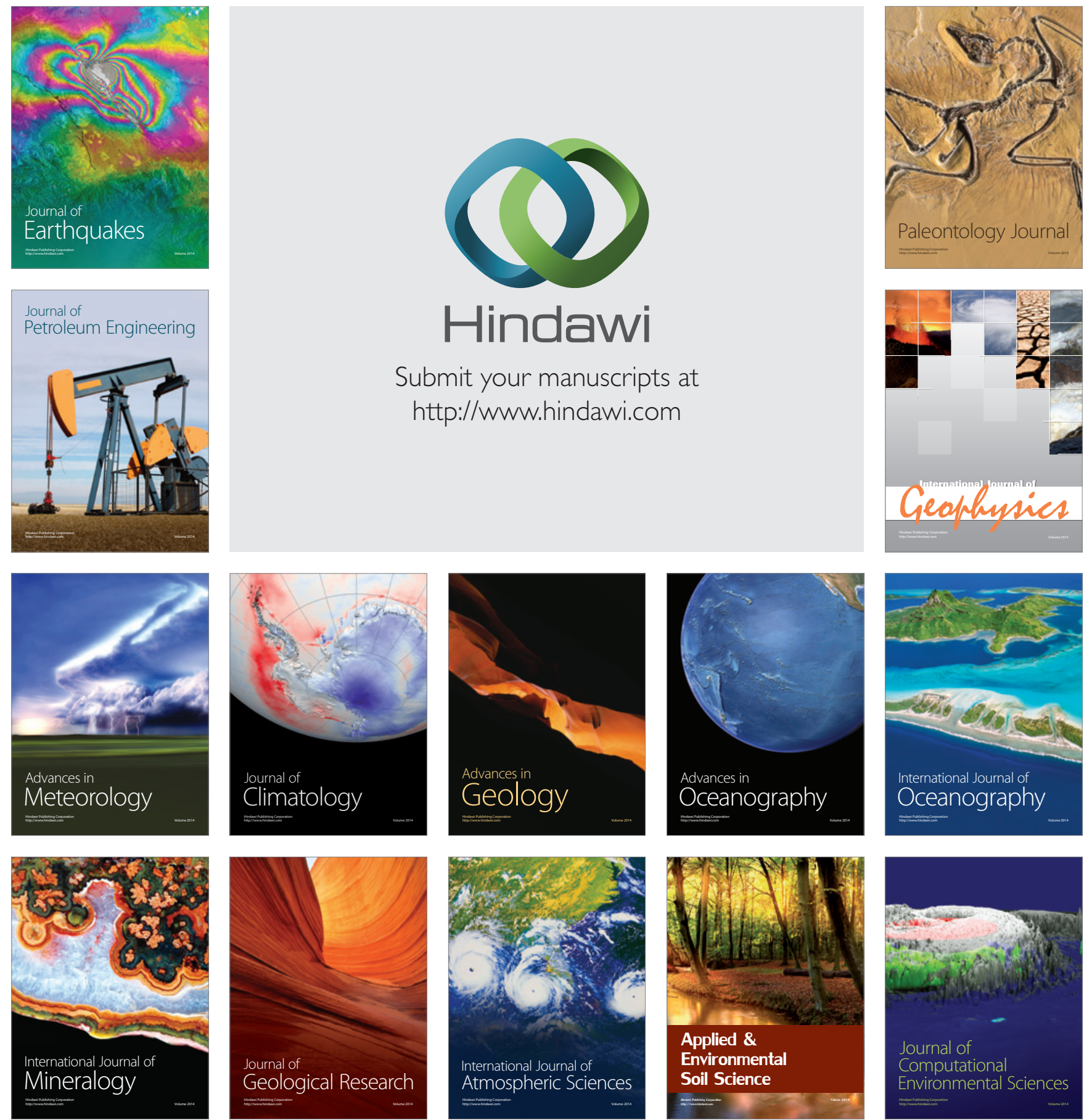